\title{
Behandeln, Aufarbeiten und Reparieren durch Kunst: zeitgenössische Darstellungen der vom Krieg „verletzten“ Landschaften Ost-Frankreichs
}

\section{Susanne Müller}

Université de Lorraine

\section{Abstract}

Der erste Teil des Beitrags ist theoretischen Aspekten des Behandelns, Aufarbeitens und Reparierens durch Kunst gewidmet. Das deutsche Verb „behandeln“ beleuchtet hierbei am deutlichsten die Vielschichtigkeit eines künstlerischen Ansatzes, der Kreation und Rezeption stets zusammendenkt, ohne die materielle bzw. „handwerkliche“ (be-hand-eln) Dimension von Kunstwerken zu missachten. Das im französischen Kontext gebrauchte Verb „reparieren“ (réparer) unterstreicht hingegen die paradoxe Wiedergutmachung, die Kunst möglich macht, ohne die Spuren (Narben) des Zerbrochenen/Verletzten seiner Inhalte zu beseitigen. Die „Aufarbeitung“ hebt schließlich hervor, dass es sich um ein Hervorholen vergangener Sujets handelt, die indes im Hier und Jetzt physische und psychische Spuren hinterlassen haben. Die vom Krieg und diversen Grenzverschiebungen gezeichneten Landschaften Ost-Frankreichs stehen im Fokus des zweiten Teils, der anhand ausgewählter zeitgenössischer Kunstwerke - insbesondere aus dem Bereich der künstlerischen Fotografie - das besondere Potenzial der Kunst im Kontext des Gedenkens des Ersten Weltkriegs hervorhebt.

La première partie s'intéresse aux aspects théoriques du « traitement », de la " Aufarbeitung » (que l'on peut traduire approximativement par « étude/appréhension critique » selon P. Laveau) et de la " réparation » à travers l'art. C'est le verbe allemand " behandeln » qui éclaire le mieux la complexité d'une approche artistique associant constamment création et réception, sans négliger la dimension matérielle et artisanale/manuelle (la main, " Hand », étant à l'origine du mot « be-hand-eln ») de l'œuvre d'art. En revanche, "réparer », verbe utilisé dans le contexte francophone, souligne la « Wiedergutmachung » paradoxale que permet l'art dont le fond n'enlève pas les traces (cicatrices) de ce qui fut blessé. Enfin, la « Aufarbeitung » indique qu'il s'agit de faire ressortir des sujets du passé, lesquels ayant laissé des traces physiques et psychiques dans le présent. Les paysages de ce qu'on nomme aujourd'hui Grand Est, marqués par la guerre et divers mouvements de frontière, se trouvent au centre de la seconde partie, qui met en avant, à travers un choix d'œuvres contemporaines - issues notamment de la photographie plasticienne -, le potentiel singulier de l'art dans le contexte de la commémoration de la Première Guerre mondiale.

The first part of this contribution is dedicated to theoretical aspects of treatment, reappraisal and reparation through the arts. The verb "(to) treat" (behandeln in German) best reflects the complexity of an artistic approach which associates creation and reception without neglecting the material or artisanal/handmade dimension (cf. the German word be-hand-eln that refers etymologically to what is taken in one's hand). "To repair", used in the francophone context, highlights the paradoxical compensation (Wiedergutmachung in German) that art makes possible without disposing the traces (scars) of what has been broken/wounded. At last, the term "reappraisal" (Aufarbeitung) emphasizes the idea of digging out former subjects, which have left, however, physical and psychological traces in the here and now. The second part focuses on Eastern France's landscapes marked by the war and various rearrangements of borders. The special potential of the arts, in the context of the commemoration of World War I, is stressed on the basis of chosen works of contemporary art, particularly from the field of artistic photography.

\section{Keywords}

Aufarbeitung $•$ zeitgenössische Kunst $\bullet$ Fotografie $・$ Kriegslandschaft $•$ Ost-Frankreich

Réparation • art contemporain • photographie plasticienne $\bullet$ paysage de guerre $\bullet$ Grand Est

Reappraisal $\bullet$ contemporary art $\bullet$ artistic photography $\bullet$ war landscape $\bullet$ Eastern France

\section{EINLEITUNG}

Ausgehend von den drei im Titel erwähnten Verben Be-hand-eln, Aufarbeiten, Reparieren - interessiert sich der erste Teil meines Beitrags für die soziale Funktion von Kunstwerken, jenseits einer dezidiert „sozial engagierten“ Kunst wie 
sie in den 70er Jahren als Antwort auf die gesellschaftlichen Umwälzungen nach 1968 typisch war. ${ }^{1}$ Vielmehr steht mein Ansatz in der Tradition des von Joseph Beuys zeitgleich entworfenen Begriffs der „sozialen Plastik“, demzufolge die Kunst auf die Gesellschaft verändernd einwirken soll. Der Beuys'sche „erweiterte Kunstbegriff“ schließt dabei auch kreatives Handeln im Alltag ein („Jeder Mensch ist ein Künstler“, proklamierte Beuys $1978^{2}$ ), was eine strikte Grenztrennung zwischen Kunst und Nicht-Kunst unmöglich macht. Kunst definiert sich nach Beuys nicht durch kunstinhärente ästhetische Kriterien, sondern vielmehr durch ihr Potenzial, auf originellem Wege gesellschaftliche Veränderungen in Gang zu bringen. Dies kann auch durch die Lehre geschehen. Als Beuys 1973 in seinem Düsseldorfer Atelier die „Freie Internationale Hochschule für Kreativität und interdisziplinäre Forschung" eröffnete - es handelte sich dabei um einen Verein, der bis 1988, also zwei Jahre über Beuys' Tod hinaus Bestand hatte -, verkündete er: „Lehrer zu sein, ist mein größtes Kunstwerk."3 Die Übermittlung von Wissen und Erfahrungen, wie sie die Lehre kennzeichnet, lässt sich auch als ein Bestandteil von Kunst begreifen, die sich mit geschichtlichen Aspekten der Wirklichkeit auseinandersetzt. Es lässt sich dabei ein Bogen spannen vom Genre Historienmalerei, das seinen Ursprung in der Renaissance und seinen Höhepunkt im 17. und 18. Jahrhundert hatte, über die Arbeiten der künstlerischen Moderne, die explizit den Ersten Weltkrieg behandeln, die Strömungen der zweiten Hälfte des 20. Jahrhunderts, die sich vor allem ab den 1970er Jahren dem Zweiten Weltkrieg und den anschließenden gesellschaftlichen Umwälzungen nähern, und heutigen künstlerischen Arbeiten, welche die aktuellen politischen und wirtschaftlichen Krisen und Konflikte behandeln oder auf neue Weise die Vergangenheit beleuchten und dabei stets auch der Überlieferung dienen.

Diese Betonung der sozialen oder im weiteren Sinne politischen Dimension von Kunst, die mit der Befragung der gesellschaftlichen Wirklichkeit - und also auch ihrer Vergangenheit - einhergeht, ist in der Tat heute erneut aktuell. Der britischen Kunsthistorikerin Claire Bishop zufolge vollzieht die zeitgenössische Kunst einen „social turn“ (Bishop 2006), also eine Wende hin zu sozialen, ethischen Themen und Fragestellungen, häufig im Kontext partizipativer Projekte, die soziale (häufig marginalisierte) Gruppen in die künstlerische Investigation aktiv einbeziehen und hierdurch die von Bourriaud postulierte relationale Ästhetik (Bourriaud 1998) um den Aspekt eines kollaborativen Kunstschaffens erweitert, das die soziale Dimension als solche thematisiert.

\footnotetext{
Als Beispiel seien hier die feministischen Body-Art-Performances erwähnt, etwa von Valie Export, die 1968 mit ihrem „Tast- und Tappkino“ für Furore sorgte.

2 Dieses Zitat, häufig falsch ausgelegt, sollte in seinem Kontext gelesen werden: „Jeder Mensch ist ein Künstler. Damit sage ich nichts über die Qualität. Ich sage nur etwas über die prinzipielle Möglichkeit, die in jedem Menschen vorliegt [...] Das Schöpferische erkläre ich als das Künstlerische, und das ist mein Kunstbegriff." (aus dem Vortrag "Jeder Mensch ein Kunstler - Auf dem Weg zur Freinen am 23. Mäz 1978 im Humbold-Haus des Internationales Kulturzentrums Achberg INKA), zitie nach Beuys (2000), in Beuys, Eva (Hg.), Das Geheimnis der Knospe zarter Hülle. Texte 1941-1986, München, Schirmer Mosel.

3 Zitiert nach Florian Malzacher (2015), „Nützliche Kunst: welche Rolle kann Kunst in der Politik spielen?" [https:/www.goethe.de/de/kul/tut/gen/tup/20559574.html, Zugriff: 04.02.2021].
}

Insbesondere seit den politischen Umwälzungen nach 1989 lässt sich von einer globalisierten postkolonialen Kunst sprechen, in der nicht selten die Kunstwerke (zumindest auch) die Funktion erfüllen, die Missverhältnisse im Herkunftsland des Künstlers aufzuzeigen und anzuprangern. Man mag in diesem Kontext an die wegbereitende Documenta 11 aus dem Jahre 2002 denken, deren Kurator Okwui Enwezor eine gezielt internationale Perspektive mit zahlreichen sozialpolitischen Aktionen und Werken anbot. Einer sozial orientierten Kunst liegt schließlich die Auffassung der Untrennbarkeit von Kreation (Herstellen von Kunstwerken) und Rezeption (Wirkung von Kunstwerken) zugrunde. Im Deutschen kann man hier auf den gemeinsamen etymologischen Ursprung von „Werk" und „Wirken“ hinweisen. Wer Kunst schafft, wirkt auf andere ein, wenn diese Anderen nicht gleich selbst in den Schaffensprozess einbezogen werden, wie es Joseph Beuys und Guy Debord bereits vor fünfzig Jahren gefordert haben. Jenseits einer passiv-kontemplativen Haltung wird der Zuschauer somit zum aktiven Teilnehmer, der seinerseits unter dem Eindruck der Werke gestalterisch auf die Welt einwirkt. Nach einer kurzen Definition und Einordnung der Verben „behandeln“, „aufarbeiten“ und „reparieren“ im Spannungsfeld von Kunst und Psychoanalyse gehe ich im zweiten Teil dieses Artikels der Frage nach, auf welche Weise und in welcher Absicht Kunst sich mit einer potenziell traumatischen Vergangenheit beschäftigen kann, einer Vergangenheit also, die durch politische Konflikte und kriegerische Auseinandersetzungen geprägt ist, welche die gesellschaftliche Realität bis heute nachhaltig - wenn auch teils auf unbewusste Weise beeinflussen. Die zunächst folgenden theoretischen, begriffsklärenden Bemerkungen gelten dabei für sämtliche (Kriegs-) Traumata, die „Narben“ in den Seelen, Körpern, Architekturen und Landschaften hinterlassen haben. Die anschließenden Beispiele, insbesondere aus dem Bereich der zeitgenössischen Kunst-Fotografie, behandeln und bearbeiten explizit die Spuren des Ersten Weltkriegs in den Landschaften Ost-Frankreich. Den ausgewählten (Foto-)Arbeiten gelingt es, den Krieg nicht nur zu repräsentieren, also darzustellen oder zu dokumentieren (was zweifelsohne durch den zeitlichen Abstand erschwert wird), sondern inn in seiner Eigenschaft als Vergangenes präsent werden zu lassen, das heißt inn zu vergegenwärtigen (siehe die französische Unterscheidung zwischen présentifier und représenter) und ins heutige Bewusstsein zu holen. Nicht die vergangenen Ereignisse werden als solche abgebildet oder befragt, sondern vielmehr der Blick, den wir Heutigen auf diese Vergangenheit richten.

\section{BEHANDELN,AUFARBEITEN,REPARIEREN}

\section{Behandeln}

Das deutsche Verb „behandeln“ hat drei Bedeutungen. Erstens: medizinisch behandeln zwecks Besserung, Heilung eines Leidens (französisch soigner); zweitens: mit jemandem/etwas umgehen (französisch traiter); drittens: etwas 
bearbeiten, mit den Händen oder intellektuell (französisch manier, manipuler). Die ursprüngliche, etymologische Bedeutung ist: „in die Hand nehmen, nach etwas greifen“4. Im Kunstwerk verstanden als Prozess und Resultat des künstlerischen Schaffens - sind diese drei Aspekte der Behandlung präsent: die manuelle, handwerkliche Bearbeitung und Formung eines Materials, die Behandlung eines Themas oder einer Fragestellung und schließlich auch die Behandlung im Sinne einer psychischen Einwirkung, die, wenn sie auch nicht direkt „heilt“ oder „Besserung“ verschafft, so doch beim Betrachter psychische Veränderungsprozesse in Gang bringen kann. Dies kann geschehen durch affektive Katharsis ${ }^{5}$ (Ausleben, Abreagieren), die Sublimierung ${ }^{6}$ von partiell destruktiven Trieben, wie sie eine wesentliche Rolle in Freuds kunsttheoretischen Schriften einnimmt, oder durch die Bewusstwerdung verdrängter Erinnerungen, die eine intellektuelle Weiterbearbeitung möglich machen. Insbesondere diesem letzten Punkt kommt dabei eine wesentliche Bedeutung für die in der Einleitung erwähnte soziale bzw. gesellschaftlich-politische Funktion von Kunst zu. Die Behandlung des Krieges als künstlerisches Sujet, z. B. in Form der Behandlung eines fotografischen Materials, das auf Ansichten heutiger Landschaften basiert, geht somit idealerweise mit der Behandlung der Betrachter einher, der Behandlung derjenigen also, die sich auf die Befragung des Krieges durch den Künstler einlassen und seinen Blick auf das Vergangene von heute aus nachvollziehen. Die Mehrdeutigkeit und Offenheit gerade künstlerischer Fotografien erlaubt zudem eine Verknüpfung mit eigenen Erfahrungen, die ihrerseits einer neuen Befragung und Behandlung zugänglich werden.

\section{Aufarbeiten}

Die Aufarbeitung, noch schwerer ins Französische zu übertragen als die mehrdeutige Behandlung, knüpft an die eben erwähnte Bewusstwerdung oder -machung von Themen an, die aufgrund ihres traumatischen Potenzials oder der Schuldund Schamgefühle, die mit innen verbunden sind, wenn nicht verdrängt, so doch einer einfachen Bearbeitung unzugänglich sind. Sie bedürfen quasi einer Durcharbeitung ${ }^{7}$, also einer wiederholten Verbalisierung oder einer anderen Form der Bearbeitung, etwa im künstlerischen Ausdruck. Die Kunst erlaubt Formen der Arbeit, die vielleicht am ehesten mit der von Freud sogenannten „Traumarbeit“ zu vergleichen sind: Diese produziert den manifesten (erinnerbaren und erzählbaren) Trauminhalt auf der Basis der latenten Traumgedanken, die unbewusst bleiben, sofern die Traumdeutung nicht zu innen

4 Vgl. https://www.dwds.de/wb/etymwb/behandeln [Zugriff: 04.02.2021].

5 Seinen Ursprung hat der Begriff der Katharsis in der aristotelischen Poetik. Es handelt sich, bezogen auf die griechische Tragödie, um eine „Reinigung“ von Affekten, die der Zuschauer durch das Durchleben von Rührung und Schauder erfährt.

6 Sublimierung nach Freud bezeichnet den Vorgang der Umleitung von Energie aus sexuellen und aggressiven Trieben hin zu künstlerischen oder intellektuellen, also gesellschaftlich anerkannten Interessen. In Die ,kulturelle " Sexualmoral und die moderne Nervoschaftlich anerkannten Interessen. In Die „kulturelle "Sexualmoral und die moderne Nervo-
sität (1908) liefert Freud eine erste Definition der Sublimierung, die indes in seinen Schriften keine eigentliche Theoretisierung erfährt: „Er [der Sexualtrieb] stellt der Kulturarbeit außerordentlich große Kraftmengen zur Verfügung, und dies zwar infolge der bei ihm besonders ordentlich große Kraftmengen zur Verfügung, und dies zwar infolge der bei inm besonders ausgeprägten Eigentümlichkeit, sein Ziel verschieben zu können, ohne wesentlich an Intensität abzunehmen. Man nennt diese Fähigkeit, das ursprünglich sexuelle Ziel gegen ein anderes, nicht mehr sexuelles, aber psychisch mit ihm verwandtes, zu vertauschen, die Fähigkeit zur Sublimierung."

7 Vgl. Freuds Aufsatz Erinnern, Wiederholen und Durcharbeiten von 1914. vorstößt und die Traumarbeit quasi rückgängig macht. Der sich in der Traumarbeit vollziehende „Übersetzungs”-Prozess ist mit jener Übertragung vergleichbar, die sich im Kunstwerk hinsichtlich der vor- oder unbewussten Beweggründe des Künstlers ausdrückt. Anders als durch eine bewusste Versprachlichung (etwa durch eine intellektuelle Analyse) gelingt es Kunstwerken, Inhalte zu repräsentieren, die auf individueller oder kollektiver Ebene bislang keiner Aufarbeitung zugänglich sind, wie etwa traumatische Kriegserinnerungen. Nicht zufällig wird die Kunst - jegliche Kunstform eingeschlossen - somit häufig zum Vorreiter hinsichtlich des Aufbrechens oder Aufkeimens von gesellschaftlichen Krisen und Konflikten. ${ }^{8}$ Sie kann somit den Grundstein für eine weitergehende Aufarbeitung legen.

\section{Reparieren}

1950 schrieb der französische Poet Francis Ponge, dass es an den Künstlern sei, die Welt in ihren Ateliers zu reparieren. ${ }^{9}$ Die Vorstellung, dass Kunst zu einer „Reparation“ (im symbolischen, psychischen Sinne) beitragen kann, wird in letzter Zeit verstärkt, insbesondere im französischsprachigen Kontext diskutiert, nicht selten im Kontext der komplexen Beziehungen zwischen Frankreich und seinen ehemaligen Kolonien und der in diesem Zuge unter wissenschaftlichem Deckmantel erbrachten Beutekunst. ${ }^{10}$ Ein theoretischer Ursprung ist in der reparation (auf Deutsch mit „Wiedergutmachung" übersetzt) nach der britischen Psychoanalytikerin Melanie Klein zu sehen, der zufolge die Reparation - bei Klein Wiedergutmachung bzw. Annullierung der gegen das geliebte Objekt gerichteten destruktiven Triebe und Fantasien - die Grundlage der schöpferischen Tätigkeit darstellt. In der Kunst geht es indes nicht um eine Wiedergutmachung oder Wiederherstellung (weshalb ich es vorziehe, den Begriff der „Reparation" beizubehalten). Ich zitiere André Louis-Paré, der die These des französischen Kunstwissenschaftlers Jacinto Lageira zusammenfasst:

„Die künstlerische Reparation ist eine materielle und symbolische Operation“. Es handelt sich nicht um eine Wiederherstellung - man kann nicht wiederholen, was vergangen ist -, sondern vielmehr um ein neues Verständnis der Geschichte, wie sie faktisch erzählt und von nun an assoziiert wird mit der Fiktion, dem Begehren, sie anders zu erzählen. ${ }^{11}$

8 In Bezug zum Thema dieses Artikels lassen sich als Beispiele Werke der europäischen Kunst der unmittelbaren Nachkriegszeit, sowohl des Ersten wie des Zweiten Weltkriegs, anführen. In beiden Fällen waren es Künstler, die durch ihre Werke das Unsagbare und anfuhren. In beiden Fâllen waren es Künstler, die durch inre Werke das Unsagbare und Nichtrepräsentierbare der Kriegsgreuel auszudrücken vermochten. In der bildenden Kuns lassen sich hier beispielhaft für den Ersten Weltkrieg Max Beckmann und Otto
für den Zweiten Weltkrieg Georg Baselitz, Anselm Kiefer und Joseph Beuys.

9 Das Originalzitat findet sich in Le murmure. Condition et destin de l'artiste von 1956: "La fonction de l'artiste est fort claire : il doit ouvrir un atelier, et y prendre en réparation le monde, par fragments, comme il lui vient."

10 Als exemplarisches Kunstwerk, das die schwierigen Beziehungen zwischen Frankreich und seinen ehemaligen afrikanischen Kolonien zum Thema hat, sei hier auf Kader Attias Installation The Repair: from occident to extraoccidental cultures aus dem Jahr 2012 hingewiesen. 11 „Selon le philosophe de l'art Jacinto Lageira, „la réparation artistique est une opération matérielle et symbolique“. Elle n'est pas une restauration - on ne peut refaire ce qui a eu lieu -, elle propose plutôt une nouvelle compréhension de l'histoire telle qu'elle est rapportée dans les faits et, désormais associée à la fiction, au désir de raconter autrement.", zitiert nach https://www.ledevoir.com/opinion/idees/521917/pour-une-reparation-symbolique-a-travers-l-art [Zugriff: 04.02.2021]. Alle nachfolgenden Zitate im Text wurden von der Autorin übersetzt. 


\section{DER ERSTE WELTKRIEG IN DER ZEITGE- NÖSSISCHEN KUNST(-FOTOGRAFIE) AM BEISPIEL DER LANDSCHAFTEN OST-FRANKREICHS}

Die vielerorts und in diverser Form durchgeführten Gedenkveranstaltungen der nunmehr hinter uns liegenden Jahre 20142018 haben bewiesen, dass das Gemahnen und Erinnern an den Ersten Weltkrieg nicht zwangsläufig einer trockenen Geschichtslektion ähneln muss. Das reichhaltige kulturelle Programm hat dabei in besonderem Maße gezeigt, dass die Verbindung des Vergangenen mit heutigem Erleben möglich ist und dass sich ferner die Erinnerung an eine schmerzhafte, hässliche Vergangenheit mit ästhetischem Genuss verbinden lässt. Wie im ersten Teil erwähnt, geht die künstlerische Bearbeitung eines Themas in der Tat über eine Darstellung oder gar Abbildung ihres Gegenstandes hinaus, insbesondere wenn dieser Gegenstand letztlich nicht repräsentierbar ist, sei es, weil die darstellenden Bilder emotional unerträglich sind oder durch ihre scheinbare Banalität - zu oft gezeigt (mediatisiert), zu bekannt, zu sehr anderen Kontexten verhaftet keine wahrhafte Auseinandersetzung (mehr) mit sich bringen. Die Erinnerung an den Ersten Weltkrieg ist eng mit dem Osten Frankreichs bzw. der deutsch-französischen Grenzregion verknüpft, trafen doch hier die beiden Erbfeinde Deutschland und Frankreich aufeinander. Und es sei daran erinnert, dass das Departement Moselle mit seiner Hauptstadt Metz und das Elsass seit dem Deutsch-Französischen Krieg von 1870-71, dessen bedeutendste Schlacht in Gravelotte unweit von Metz stattfand, deutsch waren und erst nach dem Ersten Weltkrieg erneut Frankreich zufielen. Hitler annektierte dann die gleichen Gebiete von 1940 bis 1945 ein weiteres Mal, was ein Trauma darstellt, das bis heute in diesen Regionen das Gedenken an die sogenannte „erste Annexion“ (1871-1918), welche wirtschaftlich und infrastrukturell durchaus positive Konsequenzen zur Folge hatte, überschattet. Selbstverständlich konzentrierten sich die Gedenkveranstaltungen im Grand Est auf Verdun und seine beeindruckenden Schlachtfelder, die nach wie vor weite Landschaftsareale prägen. Wer heute das lothringische Departement Meuse durchquert, wird allerorts an den Krieg erinnert: Die dünn besiedelte Landschaft ist nach wie vor durch Krater und spärlich bewachsene Grünflächen gekennzeichnet. Auch hundert Jahre nach den blutigen Schlachten von 1916 sind die Böden von den Giftstoffen und Schwermetallen der Waffen verseucht, was eine landwirtschaftliche Nutzung unmöglich macht und eine eigenartige Flora resistenter Pflanzen hervorgebracht hat, vor allem in der sogenannten Roten Zone, die kurz nach dem Krieg einer Mondlandschaft glich. Und auch wer nicht Halt macht und sich bewusst den Spuren des Krieges nähert, kommt kaum an der Erinnerung an den Weltkrieg vorbei, denn selbst die Schilder, die die Autoroute de l'Est auf dem Weg von Metz nach Paris säumen, wecken düstere Assoziationen: Douaumont, Les Éparges... Ceux de $14^{12}$ werden zu gespenstischen Weggefährten.

\footnotetext{
12 Dies der Titel der kritischen Kriegserzählungen von Maurice Genevoix aus dem Jahr
} 1950. Deutsch: Die von 14.
Diese unmittelbare Vergegenwärtigung des Vergangenen, wie es das erwähnte Buch von Maurice Genevoix erlaubt, gelingt auch Werken der visuellen Kunst auf teilweise beeindruckende Weise, steht hier doch die sinnliche, ästhetische Erfahrung im Vordergrund des Rezeptionsprozesses. Wie eingangs erwähnt, interessiere ich mich insbesondere für zeitgenössische Werke. Zu den gleich vorgestellten Beispielen neuerer Fotografie, die den ersten Weltkrieg im Osten Frankreichs - insbesondere in der Gegend von Verdun - thematisiert, seien vorweg folgende Fragen aufgeworfen: Wie lässt sich die düstere Vergangenheit des Ersten Weltkriegs in eine visuelle Sprache übersetzen, die vom heutigen Publikum verstanden wird? Wie lässt sich die Erinnerung bewahren an Gräueltaten eines Krieges, dessen letzte Zeitzeugen mittlerweile verstorben sind, ${ }^{13}$ und die daher möglicherweise fern oder gar abstrakt wirken? Wie lässt sich die Erinnerung bewahren und zugleich in ihren Formen des gemeinschaftlichen/gesellschaftlichen Gedenkens (französisch commémoration) erneuern und in der Gegenwart verankern? Wie kann die heutige Kunst zum kollektiven Gedächtnis jenseits von Militärparaden und Denkmälern beitragen, die nur mehr eine kleine Minderheit der Bevölkerung ansprechen? Können Künstler oder Fotografen schließlich die Rolle von Historikern einnehmen, wie diverse Publikationen der letzten Jahre es anregen? ${ }^{14}$

Eine Antwort auf diese letzte Frage mag in den Hypothesen des deutschen Kunsthistorikers Aby Warburg, einem Zeitzeugen des Ersten Weltkriegs, zu finden sein: Er geht von einem „Nachleben“ im Sinne eines Fortwirkens der Bilder aus, die das Potenzial besitzen, die Gegenwart zu durchkreuzen und zu befragen. Die Menschheitsgeschichte und damit auch die Kunstgeschichte wird von inm nicht als linear, sondern dialektisch und anachronistisch aufgefasst, wie es Georges Didi-Hubermann in seinem Warburg gewidmeten Werk L'image survivante: histoire de l'art et temps des fantômes selon Aby Warburg (2002) ${ }^{15}$ hervorhebt. Das Nachleben der Bilder schließt dabei auch die Wiederkehr des Verdrängten ein und knüpft damit an die „Aufarbeitung“ durch das besondere Potenzial von Kunstwerken an. Der (künstlerischen) Fotografie, die per se die Zeit als solche befragt und das Vergangene heraufbeschwört, wie es das berühmte ça-a-été von Roland Barthes postuliert, kommt hierbei eine besondere Rolle zu, gelingt es ihr doch, die visuellen Spuren der Vergangenheit im heutigen Kontext zu verankern und auf die Ambiguitäten hinzuweisen, die hierdurch entstehen.

Dass die folgenden Beispiele sich auf Landschaftsfotografie fokussieren, ist nicht nur dem Umstand geschuldet, dass diese das vorherrschende Genre in der fotografischen Annäherung an den Ersten Weltkrieg darstellt. Landschaft, sowohl ästhetischer wie auch geographischer Begriff, bezeichnet gemeinhin den sichtbaren Ausschnitt der Umgebung, der somit stets vom Blick des Betrachters abhängt, insofern als er von

13 Der letzte Veteran des Ersten Weltkrieges, Lazare Ponticelli, verstarb am 12. März 2008 .

14 Beispielhaft erwähnt sei hier Jacinto Lageiras Buch L'Art comme Histoire, Un entrelacement de poétiques von 2016.

15 In der deutschen Übersetzung von 2010: Das Nachleben der Bilder. Kunstgeschichte und Phantomzeit nach Aby Warburg. 
inm konstruiert wird. Die Landschaft ist daher immer bereits kulturell geprägt, da sie menschliche Spuren trägt (und sei es nur als Resultat eines menschlichen Blickes). Dieser Aspekt der menschlichen „Anwesenheit" selbst in den menschenleeren, öden Gegenden der ehemaligen Kriegshandlungen trägt zur Evokation des Vergangenen bei, auch wenn diese Vergangenheit materiell nur noch durch teils undefinierbare Überreste repräsentiert ist.

\section{Beispiele}

In den nun folgenden Beispielen stütze ich mich unter anderem auf die Ausstellung La Mémoire Traversée: paysages et visages de la Grande Guerre („Die durchquerte Erinnerung: Landschaften und Gesichter des Großen Krieges"), die vom 30. Oktober 2014 bis zum 18. Januar 2015 im Rahmen der Aktion „Mois de la photo“ im Pariser Kulturzentrum Eléphant Paname gezeigt wurde. Die hier ausgestellten Fotografien sprechen weniger vom Krieg als solchem, sondern vielmehr von der Erinnerung an ihn und knüpfen damit an die zuvor dargelegten theoretischen Gedanken einer Vergegenwärtigung des Vergangenen an. Inwieweit den ausgewählten Beispielen die Behandlung, Aufarbeitung oder gar Reparation gelingt, soll am Ende des Beitrags kurz erörtert werden. Jacques Grison wurde 1958 in Verdun geboren. ${ }^{16}$ Seine Fotografien lassen sich verstehen als Befragung der persönlichen Beziehung, die inn mit den vom Ersten Weltkrieg verletzten Landschaften verbindet. „Die intime Kenntnis eines Gebietes“, schreibt Laurent Loiseau, einer der Kuratoren der Ausstellung La Mémoire Traversée, „erlaubt es den Fotografen jener Landschaften, deren Kinder sie sind, deren Narben nicht nur zu nutzen, um von einem historischen Drama zu berichten, sondern ebenfalls um eine künstlerische Arbeit zu verrichten, die Zeugnis ablegt vom Umherirren des Imaginären"17 (Mois de la Photo 2014: 29). Die Fotos der Serie Verdun, 30000 jours plus tard („Verdun, 30000 Tage später") aus dem Jahr 2008 decken anhand subtiler Details auf, wie die Flora in Verdun auch fast hundert Jahre nach der Schlacht noch sichtbare Narben des Krieges trägt. Die Landschaft scheint hier selbst zum Träger der Erinnerung zu werden, eine Erinnerung, die der Blick des Fotografen quasi freilegt. Die Ausstellung Devant Verdun („Vor Verdun“), die im Arsenal Metz anlässlich der Gedenkfeiern zum 100. Jahrestag der Schlacht von Verdun organisiert wurde, zeigte verschiedene Verdun-Serien von Grison, die es den Betrachtern erlauben, den ehemaligen Kriegsschauplatz auf ästhetisch-sensible Weise neu zu erkunden. Immer wieder wird der Zuschauer dabei veranlasst, den Platz des Fotografen nachzuempfinden und seinen

16 Eindrücklich schreibt Grison zur intimen Beziehung, die er seit Geburt zum Kriegsschauplatz Verdun hat: „Ich bin in Verdun geboren, auf der Entbindungsstation, die sich

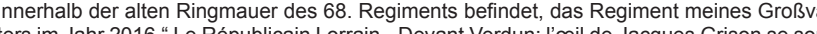
. qui se situe dans l'ancienne enceinte du 68e Régiment d'Infanterie ; le propre régiment de mon grand-père en 1916

17 Originalzitat: „La connaissance intime d'un territoire permet aux photographes de paysages dont ils sont les enfants d'utiliser leurs cicatrices non seulement pour témoigner d'un drame historique, mais également pour effectuer un travail plus plasticien rendant compte de l'errance de l'imaginaire."
Blickwinkel einzunehmen, wie in einem Foto, das den Körper des Fotografen als Schatten aufscheinen lässt. Eine paradoxe Anwesenheit, welche die Abwesenheit der Körper der Soldaten ebenso zu unterstreichen scheint, wie deren Waffen und Schuhe, die sich in den Wäldern um Verdun als resistente Überreste erweisen.

Die unheimlichen, teilweise unkenntlichen oder camouflierten Überreste der Präsenz der gefallenen Soldaten und die „Narben“, die Bomben und Schüsse der Natur zugesetzt haben, prägen auch die Arbeiten des 1932 geborenen französisch-amerikanischen Fotografen J.S. Cartier, der als Wegbereiter der jüngeren Generation von Fotografen der Schauplätze des Ersten Weltkriegs gelten kann. Sein ambitioniertes Projekt Traces de la Grande Guerre („Spuren des Großen Krieges") (1984-1994) stellt eine fotografische Bestandaufnahme der Spuren des Krieges dar, für die er weite Teile Europas bereist hat. Aus diesem Projekt ist eine vierbändige Publikation hervorgegangen, deren zweiter Teil den vergessenen Überresten in der Champagne-Ardenne, in Lothringen und im Elsass gewidmet ist.

Dem Fotografen als (physischem und psychischem) Individuum kommt im Zuge seiner Arbeit zu den Kriegsspuren, spezieller den Schlachtfeldern, eine besondere Bedeutung zu. Wie der Kurator Laurent Loiseau schreibt, ist der Akt, ein Schlachtfeld zu durchstreifen, keineswegs belanglos: „Egal, ob er Reporter oder Künstler ist, dieser engagierte Zeuge kommt niemals unbescholten aus dem Theater der Geschichte heraus. Sein Umherreisen ist eine Suche, die manchmal vergeblich und halluzinatorisch ist. Häufig ein wahrhaftes Leiden"18 (Mois de la Photo 2014: 6).

Der Blickwinkel des Fotografen, der sich „an die Stelle“ des Soldaten begibt - physisch, an den Ort des Kampfes, aber in gewisser Weise auch mental - wird wie die Fotografie selbst zum „Medium“, das es dem Zuschauer erlaubt, das Gewesene nachzuvollziehen. Jean Richardot (geboren 1959) gelingt dieser empathische Blick, indem er buchstäblich die Perspektive des Soldaten einnimmt. Sein Kameraobjektiv befindet sich hierzu am Boden, im Schnee oder Matsch, in dem einst die Körper der Soldaten verharrten oder dahinsiechten. Auch hier sind es materielle Überbleibsel des Krieges, wie etwa verrottende Munitionsreste, die zugleich das Vergangene heraufbeschwören und die Bilder im Jetzt verankern. In Zusammenarbeit mit Stéphane Audoin-Rouzeau und Gerd Krumeich ist 2008 das Buch Cicatrices („Narben“) erschienen, das diese Arbeiten zusammenfasst. In einer neueren Serie mit dem Titel Ça fait partie du paysage („Das ist Teil der Landschaft“), befragt Richardot den Status der Ruinen des Ersten Weltkriegs im Kontext der heutigen (Industrie-)Landschaften Frankreichs. Die großformatigen Panoramaaufnahmen, die im Rahmen der Gedenkfeierlichkeiten im Pariser Élysée-Palast ausgestellt wurden, zeigen Orte, deren Ambiguität häufig erst auf den zweiten Blick zutage tritt, da die Spuren des Krieges, wie der Titel besagt, derart als Bestandteil der Landschaft

18 Originalzitat: „Qu'il soit reporter ou artiste, ce témoin engagé ne ressort pas indemne du théâtre d'histoire. Sa pérégrination est une quête, parfois vaine et hallucinée. Souvent une réelle souffrance. 
erscheinen, dass ihre Fremdartigkeit und zeitliche Diskrepanz zum heutigen Kontext fast unsichtbar werden. „Indem sie es vermeidet, sich auf die alleinigen Spuren des Krieges - Graffitis, Ruinen, Schutzunterkünfte, Friedhöfe etc. - zu fokussieren“, schreibt Jean Richardot auf seiner Internetseite, „dehnt die Panoramaaufnahme den Blick auf die Simultanpräsenz des heutigen Lebensumfeldes aus und bietet so eine authentische, desakralisierte Sicht auf die Überreste des Krieges an". ${ }^{19}$

In Col de la Chapelotte (Abb. 1) bestehen diese Überreste aus unspektakulären, wenn auch unnatürlichen Furchen im Waldboden, die bedeckt von einer zarten, alles gleichmachenden Schneedecke nichts von den Greueln erahnen lassen, deren Schauplatz sie einst waren. Ohne den Titel der Aufnahme, der unweigerlich auf die schweren Gefechte verweist, die auf jenem Vogesenpass im Departement Meurthe-et-Moselle ausgetragen wurden und für 2000 französische Soldaten den Tod bedeuteten, wären diese wohl kaum als Schützengräben des Ersten Weltkriegs zu erkennen. Dass sich am gleichen Ort bereits 1870 deutsche und französische Soldaten bekämpften und dass die Gestapo 1944 denselben Pass wählte, um französische Widerstandskämpfer zu erschießen, unterstreicht eindrucksvoll, dass die Landschaft als Ausschnitt und Momentaufnahme nur einen Bruchteil ihrer eigenen Geschichte offenbart. Insbesondere dort, wo die Landschaft von Vegetation geprägt ist, zeigt sich die camouflierende Wirkung der Fauna, die mit der Zeit alles „dem Erdboden gleichmacht", womit auch ihr eine entscheidende Funktion von „Reparation“ zukommt.

Ganz anders die Aufnahme aus Wittelsheim (Elsass) (Abb. 2), die eine Industrieanlage zeigt, welche die Überreste eines deutschen Bunkers beherbergt und diesen zu eigenen Zwecken umfunktioniert hat, vermutlich da seine

19 https://www.jeanrichardot.com/index.php?id=33 [Zugriff: 04.02.2021]. Der Text is ebenfalls auf der offiziellen Seite zum Centenaire-Programm zu finden: https://www.centenaire.org/fr/autour-de-la-grande-guerre/photographie-artistique/ca-fait-partie-du-paysage-jean-richardot. Originalzitat: „En évitant de se focaliser sur les seules traces de la guerre - graffitis, ruines, abris, cimetières, ...'image panoramique élargit le regard à la présence simultané de la vie d'aujourd'hui et propose ainsi une vision authentique, désacralisée, des vestiges."
Beseitigung zu aufwändig und kostspielig gewesen wäre. Umgeben von Fässern und Baustoffen, integriert sich der klobige, fensterlose Bau problemlos in eine unwirkliche Landschaft, die, trotz der Abwesenheit von Menschen, von einer rastlosen Tätigkeit zeugt, der im Kontext von Richardots Serie Symbolcharakter zukommt, handelt es sich doch offenkundig um das Umschichten von Materialien, das Errichten und Einreißen von Bauten. Die massiven Bunker des Ersten und Zweiten Weltkriegs trotzen vielerorts den industriellen und städtebaulichen Prioritäten (man denke beispielsweise an die von den Nazis errichteten „Flaktürme“, besonders abrissresistente, große und hohe Bunkeranlagen für Flugabwehrkanonen, die bis heute das Stadtbild von Berlin, Hamburg und Wien prägen). Wie der Bunker in Wittelsheim, den erst der Blick des Fotografen als solchen sichtbar macht, zwingen sie uns, der Geschichte in der Gegenwart einen Platz einzuräumen und werden damit auch zum Mahnmal wider das Vergessen.

Bei all diesen Arbeiten ist die Grenze zwischen Dokumentarund Kunstfotografie nicht leicht zu ziehen, da sich auch dem objektiven Reporter-Blick im Falle von Kriegsschauplätzen nicht selten ein subjektiver Blickwinkel beimischt. Es lässt sich jedoch festhalten, dass in der künstlerischen Fotografie (französisch photographie plasticienne) das intime (subjektive) Moment wichtiger ist als das faktische (objektive), und - beim Betrachter - das ästhetische, sinnliche Erleben wichtiger als das rationale Erkennen. Die Fotos evozieren statt zu demonstrieren und konfrontieren in ihrer Unvollständigkeit oder Ambiguität bzw. Offenheit den Betrachter stets auch mit seiner eigenen Geschichte.

Yan Morvan, geboren 1954, liefert ein Beispiel dafür, dass eine Fotografen-Karriere beides, Reportage und künstlerische Stellungnahme, verbinden kann: Nach einer siebenjährigen Arbeit für Sipa Press und Newsweek lässt Morvan die Reportagefotografie hinter sich, um sich seinem Projekt Battlefields („Schlachtfelder“) zu widmen. Seit 2004 bereist er die Kriegsschauplätze der Welt, auf den Spuren der 250 größten Schlachten, die die Geschichte geprägt haben. Zu dieser Wende in seiner Fotografenkarriere

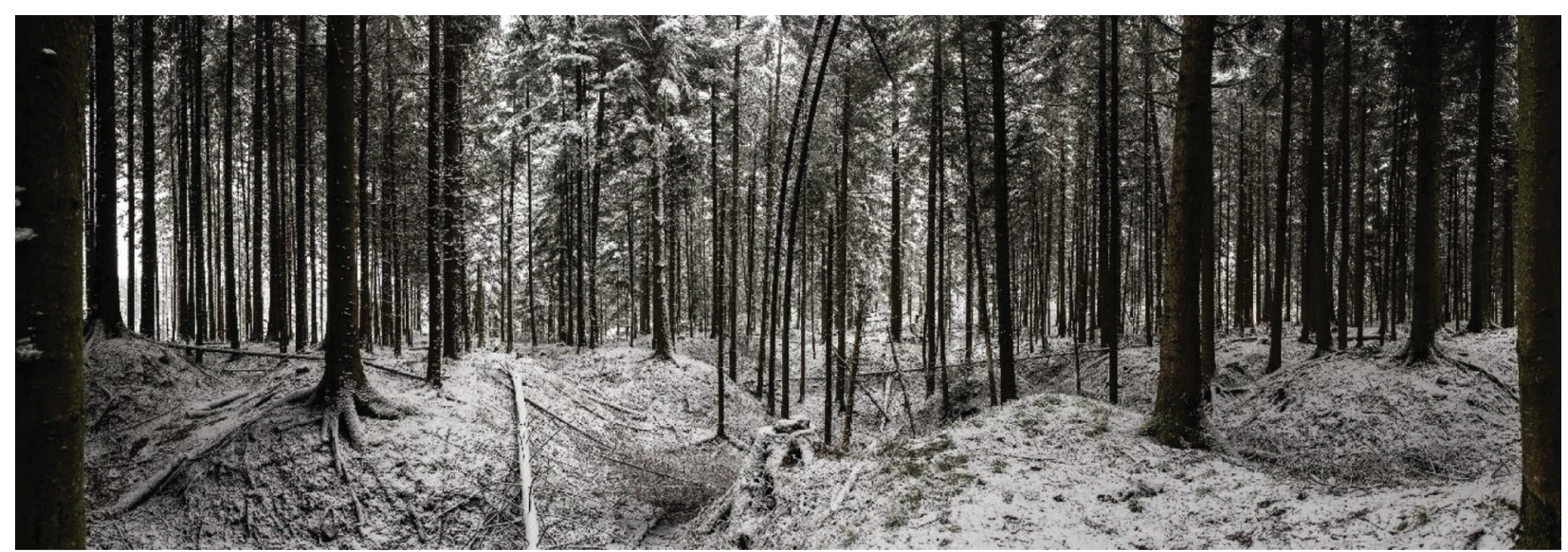

Abb.1: Jean Richardot, Col de la Chapelotte, (Forêt des Vosges où l'exploitation forestière a préservé les lignes de tranchées), de la série Ça fait partie du paysage, 2012, $140 \mathrm{~cm}$, Dibond, Assemblage panoramique. (C) Jean Richardot. 


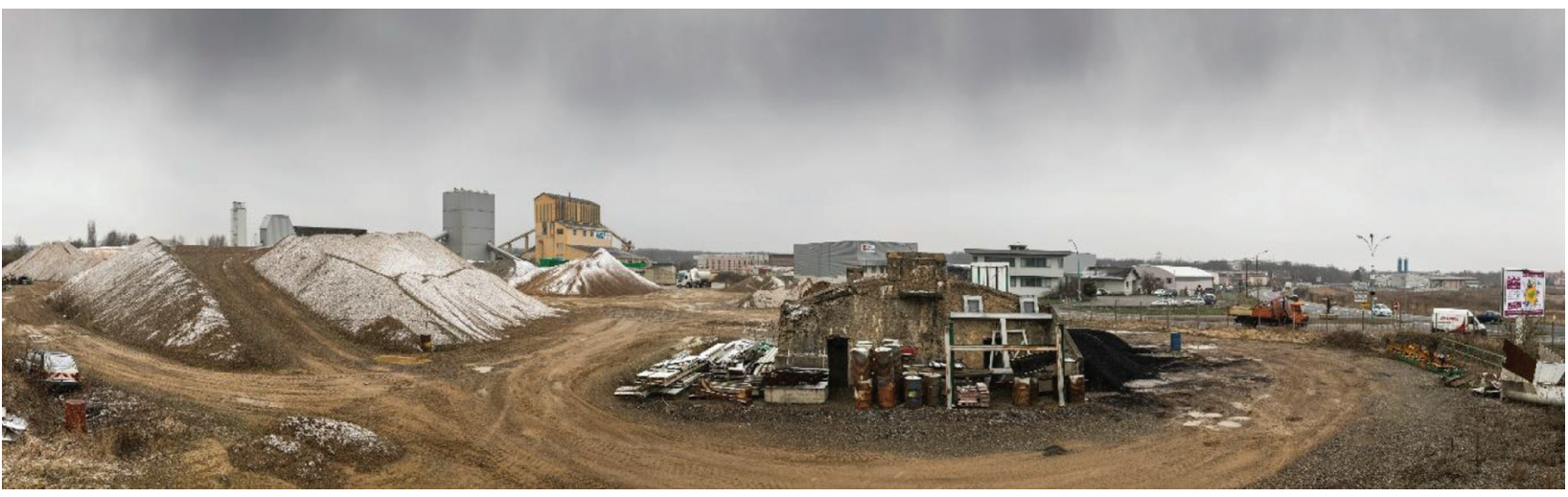

Abb. 2: Jean Richardot, La seconde vie d'un blockhaus allemand à Wittelsheim, de la série Ça fait partie du paysage, $2012,135 \mathrm{~cm}$ Dibond, Assemblage panoramique. @ Jean Richardot.

schreibt er selbst: „Ich wurde [früher] bezahlt, um einen Blickwinkel zu finden, eine Geschichte zu erzählen. Heute weiß ich, dass ich eine echte Überlegung über die Fotografie des Krieges anstelle.“ Er möchte dabei „nichts dramatisieren, nichts inszenieren." (Mois de la Photo, 2014, 21) Seine in Verdun entstandenen Aufnahmen zeigen denn auch auf schlichte und nüchterne Weise eine Landschaft, deren Kriegsnarben teilweise erst auf den zweiten Blick offenkundig werden.

Nur kurz erwähnt seien hier das 2008 erschienene Buch Les Âmes de Verdun („Die Seelen von Verdun“), das die Arbeiten der beiden Fotografen Michel Castermans (geboren 1956) und Bernard Plossu (geboren 1945) und des Autors Philippe Grasset vereint, die Serie La Terre se souvient („Die Erde erinnert sich“, ausgestellt 2003) von Jeanloup Sieff (1933-2000) sowie die Serie Fields of battle. Terre de Paix 1914-1918 („Schlachtfelder. Erde des Friedens 1914-1918“, ausgestellt 2014) des Briten Michael St Maur Sheil (Geburtsdatum leider unauffindbar). Auch diesen Arbeiten ist ein sensibler Blick auf die heutigen Landschaften der ehemaligen Kriegsschauplätze gemeinsam. Jenseits eines eindeutigen Blickwinkels auf das Vergangene laden auch diese fotografischen Projekte den Betrachter ein, einen eigenen Bedeutungshorizont zu konstruieren, den die fotografierten Landschaften lediglich andeuten.

\section{Schluss}

Die „Behandlung“ des Krieges anhand der Narben, die die Landschaften noch heute tragen, dürfte in den gezeigten Beispielen offenkundig geworden sein. Dass diese auf ihre Weise zur Aufarbeitung des Vergangenen beitragen oder zumindest den Zuschauern Stoff zum Weiterarbeiten liefern, liegt ebenfalls auf der Hand. Die Frage nach der Reparation der Kriegsnarben ist schwieriger zu beantworten: Angesichts der Fokalisierung auf die noch vorhandenen Narben der Landschaft könnte man in der Tat versucht sein zu behaupten, dass sie die Narben eher aufreißen als zu ihrer Verheilung beizutragen. Gerade das Zeigen der noch sichtbaren Narben und Überreste, die mit der Natur eine eigenartige
Symbiose eingehen, weisen jedoch darauf hin, dass hier etwas vernarbt und dem Wandel der Zeit unterworfen ist. Das Medium der Fotografie liefert somit singuläre Momentaufnahmen von Spuren eines Gestern, die morgen bereits verloren sein können. Ohne Nostalgie geht es den genannten Fotografen um eine zugleich symbolische und konkrete Thematisierung des Krieges, der unsere Lebenswirklichkeit noch heute prägt.

Die soziale Funktion dieser Arbeiten besteht in der Bewusstmachung dessen, was in den Bildern der Massenmedien, wenn überhaupt, dann meist verkitscht und ideologisch aufgeladen erscheint: eine traumatische Vergangenheit, die weltweit Wunden hinterlassen hat, deren psychische Folgen sich nicht selten von Generation zu Generation fortsetzen und durch neuere Kriege immer wieder eine Aktualisierung, jedoch selten eine Bearbeitung erfahren. Sie besteht auch darin, Bilder anzubieten, von denen aus sich Geschichte/n erzählen lässt/lassen und die der Erfahrung und dem Erleben Anhaltspunkte für eine (Über-)Mittlung bieten, die der Krieg zunächst unmöglich machte. In Der Erzähler von 1937 beschreibt Walter Benjamin die Nicht-Kommunizierbarkeit des Krieges: „Mit dem Weltkrieg begann ein Vorgang offenkundig zu werden, der seither nicht zum Stillstand gekommen ist. Hatte man nicht bei Kriegsende bemerkt, daß die Leute verstummt aus dem Felde kamen? nicht reicher, ärmer an unmittelbarer Erfahrung." (Benjamin 1996: 259) Die Kunst bietet sich als ein „Mittel“ oder Medium für die Erfahrung bzw. die Erinnerung daran an. Zudem schärft sie den Blick für das Inkongruente und Unheimliche der Lebenswirklichkeit.

Der Beitrag der Kunst zur Reparation einer traumatischen Vergangenheit kann nur ein paradoxer sein: Sie repariert das Zerbrochene, ohne seine Narben zu beseitigen. Ähnlich der Architektur integriert sie das Bestehende ins Heutige und „rekonstruiert" unter Rückgriff auf aktuelle Fragen und Anforderungen. Nur so garantiert sie die Weiterführung der Aufarbeitung und trägt zur Behandlung des Betrachters bei, die wie jene der Psychoanalyse eine unendliche ist und sich stets erneuern muss. 


\section{Bibliographie}

Audouin Rouzeau, Stéphane / Krumeich, Gerd / Richardot, Jean (2008), Cicatrices, la Grande Guerre aujourd'hui, Paris, Tallandier. Bailly, Jean-Christophe (Hg.) (2013), Les cahiers de l'Ecole de Blois $n^{\circ} 11$, „Les cicatrices du paysage“, Paris, Éditions de la Villette.

Bailly, Jean-Christophe (2011), Le dépaysement. Voyages en France, Paris, Seuil.

Benjamin, Walter (1996), „Der Erzähler“, in Ein Lesebuch, 258-284, Frankfurt am M., Suhrkamp.

Bishop, Claire (2006), „The social turn: collaboration and its discontents“, in Artforum International, 44 (6), 178-183.

Bourriaud, Nicolas (1998), Esthétique relationnelle, Dijon, Les Presses du réel.

Castermans, Michel / Grasset, Philippe / Plossu, Bernard (2010), Les âmes de Verdun. La victoire de l'homme sur la ferraille, Wavre, Éditions Mols.

Cartier J.S. / Rouaud, Jean (1994), Traces de la Grande Guerre. Les vestiges oubliés en Champagne-Ardenne, Lorraine et Alsace, Paris, Marval.

De Saint Maur, Michael (2016), Fields of battle. Lands of peace, 1914-1918, Baden, Lammerhuber.

Didi-Huberman, Georges (2002), L'image survivante: histoire de l'art et temps des fantômes selon Aby Warburg, Paris, Les éditions de Minuit.

Duroy, Stéphane / Perrot, Éric (1999), Une saison en Lorraine, Paris, Filigranes.

Jeudy, Henri-Pierre / Galera, Marie Claudia (2013), Imaginaire contemporain de la Grande Guerre, Cirey-sur-Blaise, ChâteletVoltaire.

Grison, Jacques (2016), Devant Verdun, Pomponne, Trans Photographic Press.
Freud, Sigmund (2000), „Die ,kulturelle‘ Sexualmoral und die moderne Nervosität“, Studienausgabe, IX, Frankfurt am M., Fischer Taschenbuchverlag.

Freud, Sigmund (2000), „Erinnern, Wiederholen und Durcharbeiten“, Studienausgabe, „Ergänzungsband“, Frankfurt am M., Fischer Taschenbuchverlag.

Freud, Sigmund (1987), Schriften zur Kunst und Literatur, Frankfurt am M., Fischer Taschenbuch Verlag.

Hillaire, Norbert (2019), La réparation dans l'art, Lyon, Nouvelles éditions Scala.

Jouannais, Jean-Yves (2011), Topographies de la guerre, Paris, Steidl Publishers / Le Bal.

Klein, Melanie / Rivière, Joan (1964), Love, Hate and Reparation, New York, London, Norton critical éditions (es wird hier der englische Originaltitel angeführt, da seine deutsche Übersetzung den Begriff „reparation“ auslässt [Liebe, Hass und SchuldgefühI]).

Lageira, Jacinto (2016), L'Art comme Histoire. Un entrelacement de poétiques, Paris, Éditions Mimésis.

Michel, Aurélie / Müller, Susanne (Hg). (2018), Paysage(s) de l'étrange: Arts et recherche sur les traces de l'histoire du Grand Est, Lormont, Le Bord de l'eau.

Mois de la photo (2014), La mémoire traversée: paysages et visages de la Grande Guerre, 1914-2014, Éléphant Paname / Mois de la photo à Paris 2014, Paris.

Morvan, Yan (2015), Champs de bataille, Arles, Photosynthèses.

Ponge, Francis (1956), Le murmure. Condition et destin de l'artiste, Lyon, A. Henneuse, Les Écrivains réunis.

Salber, Wilhelm (1999), Kunst, Psychologie, Behandlung, Bonn, Bouvier. Sieff, Jean-Loup (2003), La terre se souvient, Paris, Stock.

Warburg, Aby Moritz (2000), Der Bilderatlas Mnemosyne, Warnke, Martin Hrsg., Berlin, Akademie. 